Ghadeer Ghawadrah

\title{
The descriptive complexity of the family of Banach spaces with the $\pi$-property
}

Received: 25 March 2014 / Accepted: 4 August 2014 / Published online: 27 August 2014

(C) The Author(s) 2014. This article is published with open access at Springerlink.com

\begin{abstract}
We show that the set of all separable Banach spaces that have the $\pi$-property is a Borel subset of the set of all closed subspaces of $C(\Delta)$, where $\Delta$ is the Cantor set, equipped with the standard Effros-Borel structure. We show that if $\alpha<\omega_{1}$, the set of spaces with Szlenk index at most $\alpha$ which have a shrinking FDD is Borel.
\end{abstract}

Mathematics Subject Classification 46B20 - 54H05

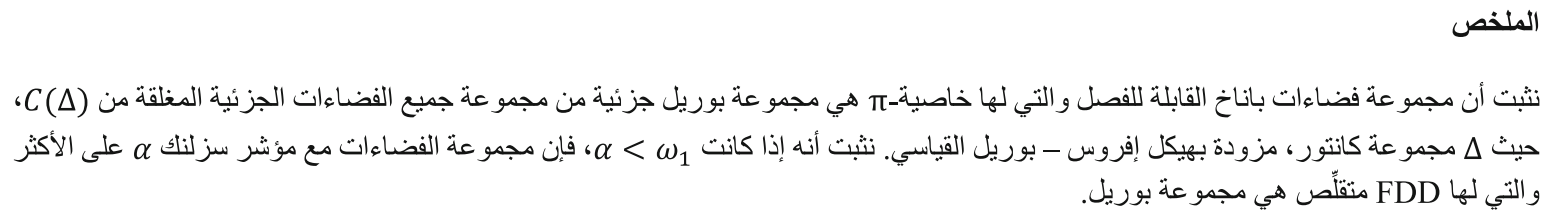

\section{Introduction}

Let $C(\Delta)$ be the space of continuous functions on the Cantor space $\Delta$. It is well known that $C(\Delta)$ is isometrically universal for all separable Banach spaces. We denote $\mathcal{S E}$ the set of all closed subspaces of $C(\Delta)$ equipped with the standard Effros-Borel structure. In [1], Bossard considered the topological complexity of the isomorphism relation and of many subsets of $\mathcal{S E}$. In addition, it has been shown that the set of all separable Banach spaces that have the bounded approximation property (BAP) is a Borel subset of $\mathcal{S E}$, and that the set of all separable Banach spaces that have the metric approximation property (MAP) is also Borel [5].

We recall that the Banach space $X$ has the $\pi_{\lambda}$-property if there is a net of finite rank projections $\left(S_{\alpha}\right)$ on $X$ converging strongly to the identity on $X$ with $\limsup _{\alpha}\left\|S_{\alpha}\right\| \leqslant \lambda$ (see [2]). We say that the Banach space $X$ has the $\pi$-property if it has the $\pi_{\lambda}$-property for some $\lambda \geq 1$.

In this note we show that the set of all separable Banach spaces that have the $\pi$-property is a Borel subset of $\mathcal{S E}$. This bears some consequences on the complexity of the class of spaces with finite-dimensional decompositions. For instance, we show that in the set of spaces whose Szlenk index is bounded by some countable ordinal, the subset consisting of spaces which have a shrinking finite-dimensional decomposition is Borel.

G. Ghawadrah ( $\square)$

Université Paris VI Case 247, 4 Place Jussieu, 75252 Paris Cedex 05, France

E-mail: ghawadrah@math.jussieu.fr 


\section{Main results}

Here is the main technical lemma.

Lemma 2.1 Suppose $\left(x_{n}\right)_{n=1}^{\infty}$ is a dense sequence in a Banach space $X$. Then, $X$ has the $\pi$-property if and only if

$\exists \lambda>1 \forall c \in\left(0, \frac{1}{4}\right) \bigcap \mathbb{Q} \forall K \forall \epsilon>0 \forall \lambda^{\prime}>\lambda \exists R \forall N \geqslant R \exists \sigma_{1}, \ldots, \sigma_{N} \in \mathbb{Q}^{R} \forall \alpha_{1}, \ldots, \alpha_{N} \in \mathbb{Q}$

$$
\begin{gathered}
\left\|\sum_{i=1}^{N} \alpha_{i}\left[\sum_{j=1}^{R} \sigma_{i}(j) x_{j}\right]\right\| \leqslant \lambda^{\prime} \cdot\left\|\sum_{i=1}^{N} \alpha_{i} x_{i}\right\| \cdot \\
\forall i \leqslant K, \quad x_{i}-\left[\sum_{j=1}^{R} \sigma_{i}(j) x_{j}\right] \| \leqslant \epsilon \\
\left\|\sum_{i=1}^{N} \alpha_{i}\left[\sum_{j=1}^{R} \sigma_{i}(j) x_{j}\right]-\sum_{i=1}^{N} \alpha_{i}\left[\sum_{t=1}^{R}\left[\sum_{j=1}^{R} \sigma_{i}(j) \sigma_{j}(t)\right] x_{t}\right]\right\| \leqslant \cdot\left\|\sum_{i=1}^{N} \alpha_{i} x_{i}\right\| .
\end{gathered}
$$

where $K, R, N$ vary over $\mathbb{N}$ and $\epsilon, \lambda^{\prime}$, and $\lambda$ vary over $\mathbb{Q}$.

Proof Indeed, suppose $X$ has the $\pi_{\lambda}$-property. Then there exists a sequence $\left(P_{n}\right)$ of finite rank projections such that $\left\|P_{n}\right\|<\lambda$, for all $n$ and $P_{n}$ converge strongly to the identity.

By perturbing $P_{n}$, we may suppose that $P_{n}$ maps into the finite-dimensional subspace $\left[x_{1}, \ldots, x_{R_{n}}\right]$ for some $R_{n}$ in $\mathbb{N}$ but we still have (2.2) and $\left\|P_{n}\right\|<\lambda$. Then, for every $N$, we may perturb $P_{n}$ slightly so that $\left\|P_{n}\right\|<\lambda$ and $P_{n}\left(x_{i}\right)$ belongs to the $\mathbb{Q}$-linear span of the $x_{j}$ for all $i \leqslant N$, such that (2.1), (2.2) and (2.3) still hold. Define now $\left(\sigma_{i}^{(n)}\right) \in\left(\mathbb{Q}^{R_{n}}\right)^{N}$, such that $P_{n}\left(x_{i}\right)=\sum_{j=1}^{R_{n}} \sigma_{i}^{(n)}(j) x_{j}$. Since $P_{n}^{2}\left(x_{i}\right)=\sum_{t=1}^{R_{n}}\left[\sum_{j=1}^{R_{n}} \sigma_{i}(j) \sigma_{j}(t)\right] x_{t}$, the three inequalities hold for all $\alpha_{1}, \ldots, \alpha_{N} \in \mathbb{Q}$, and $i \leqslant K$.

Conversely, suppose that the above criterion holds and that $\epsilon^{\prime}>0$. Pick a rational $\frac{\epsilon^{\prime}}{3 \lambda}>\epsilon>0$ and a $K$. So let $\lambda^{\prime}$ and $R$ be given as above. Then for every $N$ and $i \leqslant N$, define $y_{i}^{N}=\sum_{j=1}^{R} \sigma_{i}(j) x_{j}$, and $z_{i}^{N}=\sum_{t=1}^{R}\left[\sum_{j=1}^{R} \sigma_{i}(j) \sigma_{j}(t)\right] x_{t}$ in $\left[x_{1}, \ldots x_{R}\right]$, where the $\sigma_{i}$ are given depending on $N$. We have that

$$
\left\|\sum_{i=1}^{N} \alpha_{i} y_{i}^{N}\right\| \leqslant \lambda^{\prime} \cdot\left\|\sum_{i=1}^{N} \alpha_{i} x_{i}\right\| .
$$

for all $\alpha_{i} \in \mathbb{Q}$,

$$
\left\|x_{i}-y_{i}^{N}\right\| \leqslant \epsilon
$$

for all $i \leqslant K$, and

$$
\left\|\sum_{i=1}^{N} \alpha_{i} y_{i}^{N}-\sum_{i=1}^{N} \alpha_{i} z_{i}^{N}\right\| \leqslant c \cdot\left\|\sum_{i=1}^{N} \alpha_{i} x_{i}\right\| .
$$

for all $c \in\left(0, \frac{1}{4}\right) \bigcap \mathbb{Q}$. In particular, for every $i$, the sequences $\left(y_{i}^{N}\right)_{N=i}^{\infty}$, and $\left(z_{i}^{N}\right)_{N=i}^{\infty}$ are contained in a bounded set in a finite-dimensional space. So by a diagonal procedure, we may find some subsequence $\left(N_{l}\right)$ so that $y_{i}=\lim _{l \rightarrow \infty} y_{i}^{N_{l}}$ and $z_{i}=\lim _{l \rightarrow \infty} z_{i}^{N_{l}}$ exists for all $i$. By consequence

$$
\left\|\sum_{i=1}^{\infty} \alpha_{i} y_{i}\right\| \leqslant \lambda^{\prime} \cdot\left\|\sum_{i=1}^{\infty} \alpha_{i} x_{i}\right\| .
$$


for all $\alpha_{i} \in \mathbb{Q}$,

$$
\left\|x_{i}-y_{i}\right\| \leqslant \epsilon
$$

for all $i \leqslant K$, and

$$
\left\|\sum_{i=1}^{\infty} \alpha_{i} y_{i}-\sum_{i=1}^{\infty} \alpha_{i} z_{i}\right\| \leqslant c \cdot\left\|\sum_{i=1}^{\infty} \alpha_{i} x_{i}\right\| .
$$

for all $c \in\left(0, \frac{1}{4}\right) \bigcap \mathbb{Q}$.

Now, since the $x_{i}$ are dense in $X$, there are uniquely defined bounded linear operators $T_{K, \epsilon}: X \mapsto$ $\left[x_{1}, \ldots, x_{R}\right]$ satisfying $T_{K, \epsilon}\left(x_{i}\right)=y_{i}$ and then $T_{K, \epsilon}^{2}: X \mapsto\left[x_{1}, \ldots, x_{R}\right]$ satisfies $T_{K, \epsilon}^{2}\left(x_{i}\right)=z_{i}$ such that $\left\|T_{K, \epsilon}\right\| \leqslant \lambda^{\prime}<\lambda$ and $\left\|x_{i}-T_{K, \epsilon}\left(x_{i}\right)\right\| \leqslant \epsilon$ for all $i \leqslant K$. Let $K \rightarrow \infty$ and $\epsilon \rightarrow 0$. Then $T_{K, \epsilon}\left(x_{i}\right) \rightarrow x_{i}$ for all $x_{i} \in\left(x_{i}\right)$ strongly. Since $\left(x_{i}\right)$ is a dense sequence in $X$ and the operators $T_{K, \epsilon}$ are uniformly bounded, then $T_{K, \epsilon}(x) \rightarrow x$ for all $x \in X$ strongly. Also, lim sup $\left\|T_{K, \epsilon}-T_{K, \epsilon}^{2}\right\|=c<\frac{1}{4}$. Therefore, by [3, Theorem(3.7)], $X$ has the $\pi_{\lambda+1}$-property as $c \rightarrow 0$.

Theorem 2.2 The set of all separable Banach spaces that have the $\pi$-property is a Borel subset of $\mathcal{S E}$.

Proof Let $K, R, N$ vary over $\mathbb{N}$ and $\epsilon, \lambda^{\prime}$ vary over $\mathbb{Q}$. Let also $c \in\left(0, \frac{1}{4}\right) \bigcap \mathbb{Q}, \sigma \in\left(\mathbb{Q}^{R}\right)^{N}$, and $\alpha \in \mathbb{Q}^{N}$. Then we consider the set $E_{c, K, \epsilon, \lambda^{\prime}, R, N, \sigma, \alpha} \subseteq C(\Delta)^{\mathbb{N}}$ such that:

$$
E_{c, K, \epsilon, \lambda^{\prime}, R, N, \sigma, \alpha}=\left\{\left(x_{n}\right)_{n=1}^{\infty} \in C(\Delta)^{\mathbb{N}} ; \text { (2.1) (2.2) and (2.3) hold }\right\}
$$

This set is closed in $C(\Delta)^{\mathbb{N}}$. Therefore, for $\lambda \in \mathbb{R}$

$$
E_{\lambda}=\bigcap_{c} \bigcap_{K} \bigcap_{\epsilon} \bigcap_{\lambda^{\prime}>\lambda} \bigcup_{R} \bigcap_{N} \bigcup_{\sigma \in\left(\mathbb{Q}^{R}\right)^{N}} \bigcap_{\alpha \in \mathbb{Q}^{N}} E_{c, K, \epsilon, \lambda^{\prime}, R, N, \sigma, \alpha}
$$

is a Borel subset of $C(\Delta)^{\mathbb{N}}$. Moreover, the set

$$
E=\bigcup_{\lambda \in \mathbb{Q}} E_{\lambda}
$$

is also Borel.

There is a Borel map $d: \mathcal{S E} \longrightarrow C(\Delta)^{\mathbb{N}}$ such that $\overline{d(X)}=X$, by [8, Theorem (12.13)]. Moreover, the previous Lemma implies that

$$
X \text { has the } \pi \text {-property } \Longleftrightarrow \mathrm{d}(X) \in E \text {. }
$$

Therefore, $\{X \in \mathcal{S E} ; X$ has the $\pi$-property $\}$ is a Borel subset of $\mathcal{S E}$.

We will now prove that this result implies, with some work, that in some natural classes the existence of a finite-dimensional decomposition happens to be a Borel condition. We first consider the class of reflexive spaces. The commuting bounded approximation property (CBAP) implies the bounded approximation property (BAP) by the definition of the CBAP. By Grothendieck's theorem (see [9, Theorem 1.e.15]), the BAP and the metric approximation property (MAP) are equivalent for reflexive Banach spaces. In addition, [3, Theorem 2.4] implies that for any reflexive Banach space the CBAP is equivalent to MAP. For the set $R$ of all separable reflexive Banach spaces, Theorem (2.2), [5, Theorem 2.2] and [2, Theorem 6.3] imply that there exists a Borel subset $B=\{X \in \mathcal{S E} ; X$ has the MAP and the $\pi$-property $\}$ such that $\{X \in R ; X$ has a FDD $\}=B \cap R$.

We will extend this simple observation to some classes of non-reflexive spaces. The following result has been proved in [7]. The proof below follows the lines of [6].

Proposition 2.3 Let $X$ be a Banach space with separable dual. If $X$ has the MAP for all equivalent norms, then $X^{*}$ has the MAP. 
Proof Since $X^{*}$ is separable, there is an equivalent Fréchet differentiable norm on $X$. If $\|\cdot\|_{X}$ is a Fréchet differentiable norm and $x \in S_{X}$, there exists a unique $x^{*} \in S_{X^{*}}$ such that $x^{*}(x)=1$, and $x^{*}$ is a strongly exposed point of $B_{X^{*}}$. Since by assumption $X$ equipped with this norm has the MAP, there exists an approximating sequence $\left(T_{n}\right)$ with $\left\|T_{n}\right\| \leqslant 1$, and then for all $x^{*} \in X^{*}$ we have $T_{n}^{*}\left(x^{*}\right) \stackrel{w^{*}}{\rightarrow} x^{*}$. For all $x^{*} \in X^{*}$ which attains its norm, we have $\left\|T_{n}^{*}\left(x^{*}\right)-x^{*}\right\|_{X^{*}} \rightarrow 0$. Bishop-Phelps theorem yields that for all $x^{*} \in X^{*}$, $\left\|T_{n}^{*}\left(x^{*}\right)-x^{*}\right\|_{X^{*}} \rightarrow 0$. Therefore, $X^{*}$ has the MAP.

The set $S D$ of all Banach spaces with separable dual spaces is coanalytic in $\mathcal{S E}$ and the Szlenk index $S z$ is a coanalytic rank on $S D$ (see [1, Corollary (3.3) and Theorem (4.11)]). In particular, the set $S_{\alpha}=\{X \in$ $\mathcal{S E} ; S z(X) \leqslant \alpha\}$ is Borel in $\mathcal{S E}$ (see [8]). In this Borel set, the following holds.

Theorem 2.4 The set of all separable Banach spaces in $S_{\alpha}$ that have a shrinking FDD is Borel in $\mathcal{S E}$.

Proof Indeed, by [4, Theorem 1], we have that

$$
S_{\alpha}^{*}=\left\{Y \in \mathcal{S E} ; \exists X \in S_{\alpha} \text { with } Y \simeq X^{*}\right\}
$$

is analytic. Since the set $\{Y \in \mathcal{S E} ; Y$ has the BAP $\}$ is Borel by [5, Theorem 2.2], then

$$
G_{\alpha}^{*}=\left\{Y \in \mathcal{S E} ; \exists X \in S_{\alpha} \text { with } Y \simeq X^{*} \text { and } Y \text { has the BAP }\right\}
$$

is analytic. By [4, Proposition 7], we have that

$$
G_{\alpha}=\left\{X \in S_{\alpha} ; \exists Y \in G_{\alpha}^{*}, \text { with } Y \simeq X^{*}\right\}
$$

is analytic.

Since $\{(X, Z) ; Z \simeq X\}$ is analytic in $\mathcal{S E} \times \mathcal{S E}$ ([1, Theorem 2.3]) and $\{Z ; Z$ fails the MAP $\}$ is Borel [5], the set $\{(X, Z) ; Z \simeq X, Z$ fails the MAP $\}$ is analytic, thus its canonical projection $\{X \in \mathcal{S E} ; \exists Z \in$ $\mathcal{S E} ; Z \simeq X, Z$ fails the MAP $\}$ is analytic. Now, Proposition (2.3) implies that the set

$$
\begin{aligned}
H_{\alpha} & =\left\{X \in S_{\alpha} ; X^{*} \text { fails the } \mathrm{AP}\right\} \\
& =\left\{X \in S_{\alpha} ; \exists Z \text { with } Z \simeq X \text { and } Z \text { fails the MAP }\right\}
\end{aligned}
$$

is analytic. Since $S_{\alpha} \backslash H_{\alpha}=G_{\alpha}$ and both $G_{\alpha}$ and $H_{\alpha}$ are analytic sets in $\mathcal{S E}$, then both are Borel by the separation theorem. Now, [2, Theorem 4.9] implies that $G_{\alpha}=\left\{X \in S_{\alpha} ; X\right.$ has the shrinking CBAP $\}$. Thus,

$$
\left\{X \in S_{\alpha} ; X \text { has a shrinking FDD }\right\}
$$

is Borel by Theorem (2.2) and [2, Theorem 6.3].

Questions: As seen before, a separable Banach space has CBAP if and only if it has an equivalent norm for which it has MAP. It follows that the set $\{X \in \mathcal{S E} ; X$ has the CBAP $\}$ is analytic. It is not clear if it is Borel or not. Also, it is not known if there is a Borel subset $B$ of $\mathcal{S E}$, such that

$$
\left\{X \in S D ; X^{*} \text { has the } \mathrm{AP}\right\}=B \cap S D .
$$

This would be an improvement of Theorem (2.3). Finally, what happens when we replace FDD by basis is not clear: for instance, the set of all spaces in $S_{\alpha}$ which have a basis is clearly analytic. Is it Borel?

Acknowledgments I would like to thank my Ph.D. supervisor Gilles Godefroy at University of Paris VI for his suggestions and encouragement, and the referee for a number of helpful suggestions for improvement in the article.

Open Access This article is distributed under the terms of the Creative Commons Attribution License which permits any use, distribution, and reproduction in any medium, provided the original author(s) and the source are credited. 


\section{References}

1. Bossard, B.: A coding of separable Banach spaces, analytic and coanalytic families of Banach spaces. Fund. Math. 172, $117-157$ (2002)

2. Casazza, P.G.: Approximation properties. Handb. Geom. Banach Spaces 1, 271-316 (2001)

3. Casazza, P.G.; Kalton, N.J.: Notes on approximation properties in separable Banach spaces. Lect. Notes Lond. Math. Soc. 158, 49-65 (1991)

4. Dodos, P.: Definability under duality. Houst. J. Math. 36(3), 781-792 (2010)

5. Ghawadrah, G.: The descriptive complexity of the family of Banach spaces with the bounded approximation property. Houst. J. Math. (to appear) (2014)

6. Godefroy, G.; Saphar, P.: Duality in spaces of operators and smooth norms on Banach spaces. Ill. J. Math. 32(4), 672-695 (1988)

7. Johnson, W.B.: A complementably universal conjugate Banach space and its relation to the approximation problem Isr. J. Math. 13, 301-310 (1972)

8. Kechris, A.S.: Classical Descriptive Set Theory. Springer, Berlin (1995)

9. Lindenstrauss, J.; Tzafriri L.: Classical Banach Spaces I. Springer, Berlin (1977) 